\title{
Review Article \\ The Role of Extracellular Adenosine Generation in the Development of Autoimmune Diseases
}

\author{
F. Morandi $(\mathbb{D}),{ }^{1}$ A. L. Horenstein $\left(\mathbb{D},{ }^{2,3}\right.$ R. Rizzo, ${ }^{4}$ and F. Malavasi ${ }^{2,3}$ \\ ${ }^{1}$ Stem Cell Laboratory and Cell Therapy Center, Istituto Giannina Gaslini, 16148 Genova, Italy \\ ${ }^{2}$ Department of Medical Sciences, Laboratory of Immunogenetics, University of Torino, 10126 Torino, Italy \\ ${ }^{3}$ CeRMS, University of Torino, 10126 Torino, Italy \\ ${ }^{4}$ Department of Medical Sciences, Section of Microbiology, University of Ferrara, 44121 Ferrara, Italy
}

Correspondence should be addressed to F. Morandi; fabiomorandi@ospedale-gaslini.ge.it

Received 28 October 2017; Revised 10 January 2018; Accepted 20 February 2018; Published 26 March 2018

Academic Editor: Shin-ichi Yokota

Copyright (c) 2018 F. Morandi et al. This is an open access article distributed under the Creative Commons Attribution License, which permits unrestricted use, distribution, and reproduction in any medium, provided the original work is properly cited.

\begin{abstract}
Adenosine $(\mathrm{ADO})$ is an immunosuppressive molecule, which suppresses the immune responses by interacting with specific receptors expressed by immune effector cells. ADO is produced from ATP through the enzymatic activities of CD39 and CD73. Alternatively, $\mathrm{ADO}$ can be generated starting from $\mathrm{NAD}^{+}$, which is metabolized by the concerted action of $\mathrm{CD} 38$, CD203a/PC-1, and CD73. The role of ADO in immunity has been characterized in the last years in physiology and in pathological settings. This review examines a panel of reports focused on the functions of ADO in the context of human autoimmune/inflammatory diseases and the selected animal models. The final aim is to consider the role of adenosinergic ectoenzymes and ADO receptors as novel therapeutic targets for selected diseases.
\end{abstract}

\section{Ectonucleotidases and Adenosine}

Adenosine (ADO) is a nucleoside with pleiotropic functions, which acts as an intracellular and extracellular mediator of multiple biological processes. The concentration of $\mathrm{ADO}$ in the interstitial fluids, which is generally low, dramatically increases during tissue injury, caused by hypoxia, ischemia, inflammation, and cancer. ADO acts as a danger signal, by activating specific ADO receptors (ADOR), namely, $\mathrm{A} 1, \mathrm{~A} 2 \mathrm{~A}, \mathrm{~A} 2 \mathrm{~B}$, and $\mathrm{A} 3$, different in function and tissue distribution [1].

$A 1$ and $A 3$ receptors are coupled with $G$ proteins of the Gi, Gq, and Go family, and their activation leads to the release of calcium ions from intracellular stores. In contrast, $\mathrm{A} 2 \mathrm{~A}$ and $\mathrm{A} 2 \mathrm{~B}$ receptors are coupled with $\mathrm{G}$ proteins $\mathrm{Gs}$ or $\mathrm{Gq}$ and activate adenylyl cyclase or phospholipase C. Moreover, all adenosine receptors activate mitogen-activated protein kinase (MAPK) pathways, which include extracellular signal regulated kinase 1 (ERK1), ERK2, Jun N-terminal kinase, and p38 MAPK. ADO also has receptor-independent effects, because extracellular adenosine can cross the cell membrane and activate AMP-activated protein kinase (AMPK), adenosine kinase, and S-adenosyl homocysteine hydrolase pathways [2].

Upon interaction with these receptors, ADO can trigger different cellular responses, aimed at restoring tissue homeostasis. Among them, ADO can limit inflammatory and immune responses, to avoid tissue damage and promote the healing process [2]. Indeed, ADO acts as an immunosuppressive molecule, able to inhibit the functions of different cell populations and subsets of the immune system, including $\mathrm{T}$ and B lymphocytes, NK cells, dendritic cells, monocytes, and macrophages [3-6].

$\mathrm{ADO}$ is produced through the action of adenosinergic ectoenzymes expressed on the membrane of different cell populations. ADO may be obtained by metabolizing ATP (canonical pathway) or $\mathrm{NAD}^{+}$(alternative pathway). The canonical pathway is started by CD39, an ectonucleoside triphosphate diphosphohydrolase (NTPDase), which converts ATP to ADP. CD39 can also convert the latter molecule into AMP, fully dephosphorylated to ADO by the $5^{\prime}$-nucleotidase $\left(5^{\prime}\right.$-NT) CD73 [7]. CD39 and CD73 
have been recently proposed as novel checkpoint inhibitor targets, since $\mathrm{ADO}$ generated by these ectonucleotidases interferes with antitumor immune responses [8].

We have recently demonstrated that $\mathrm{ADO}$ can also be generated from the $\mathrm{NAD}^{+}$substrate through an alternative pathway, where CD38 (a NADase and ADP-ribosyl cyclase) plays a central role. $\mathrm{CD} 38$ converts $\mathrm{NAD}^{+}$to $\mathrm{ADPR}$, which in turn is metabolized to AMP by CD203a/PC-1 (an ectonucleotide pyrophosphatase phosphodiesterase 1). CD203a/ PC-1 can also convert ATP to AMP, which is eventually degraded to $\mathrm{ADO}$ by $\mathrm{CD} 73$, a molecule that is shared between the two pathways $[9,10]$.

ADO levels can be regulated by intracellular and extracellular mechanisms, through the action of (i) nucleoside transporters, namely, equilibrative nucleoside transporters (ENT1, ENT2, ENT3, and ENT4) and concentrative nucleoside transporters (CNT1, CNT2, and CNT3), that are able to transport ADO inside the cells $[2,11]$ and (ii) adenosine deaminase (ADA1 and ADA2), which is expressed by different cell populations and is able to convert ADO into inosine $[12,13]$. However, inosine can also induce immunosuppressive effects, through the interaction with the A2a receptor [14].

1.1. Regulatory Cells with Adenosinergic Ectoenzyme Expression. Adenosinergic ectoenzymes are present on the surface of different regulatory cell populations. $\mathrm{CD} 4^{+-}$ $\mathrm{CD} 25^{\text {high }} \mathrm{FOXP}^{+}$regulatory $\mathrm{T}$ cells (Tregs) express high levels of CD39 and CD73. The ADO produced is believed to be instrumental in abrogating the effector $\mathrm{T}$ cell functions after interacting with ADORA2A. The inhibitory effect can be counteracted by effector $\mathrm{T}$ lymphocytes through the activity of ADO deaminase (ADA). ADA, which is responsible for adenosine degradation, is hosted on CD26, a cell surface-bound glycoprotein [15]. Also, CD56 ${ }^{\text {bright }} \mathrm{CD} 16^{-}$ NK cells play multiple roles in the regulation of immune response. We recently demonstrated that $\mathrm{CD} 56^{\text {bright }} \mathrm{CD} 16^{-}$ NK cells express high levels of CD39, CD73, CD203a/PC-1, and CD157, as compared with the $\mathrm{CD} 56{ }^{\mathrm{dim}} \mathrm{CD} 16^{+} \mathrm{NK}$ subset. Moreover, CD56 ${ }^{\text {bright }} \mathrm{CD} 16^{-} \mathrm{NK}$ cells produce $\mathrm{ADO}$ and have the ability to inhibit autologous $\mathrm{CD} 4^{+} \mathrm{T}$ cell proliferation. CD38 has a central role in this process [16].

Another important regulatory cell subset is represented by $\mathrm{CD} 45 \mathrm{R} 0^{+} \mathrm{CD} 4^{+} \mathrm{CD} 49 \mathrm{~b}^{+} \mathrm{LAG}-3^{+}$type 1 regulatory $\mathrm{T}$ cells (Tr1). CD39 was recently demonstrated as promoting $\operatorname{Tr} 1$ cell differentiation by depleting extracellular ATP [17]. More importantly, CD39 is expressed on Tr1 cells and contributes to their suppressive activity through the generation of $\mathrm{ADO}$ in a discontinuous fashion. In fact, CD73, the other component of the classical pathway for $\mathrm{ADO}$ production, is expressed by responder $\mathrm{T}$ cells rather than Tr1 cells [17].

Regulatory cells are also present among B lymphocytes. A B cell subpopulation, characterized as $\mathrm{CD} 39^{+} \mathrm{CD} 73^{+}$, generates $\mathrm{ADO}$, and it is endowed with regulatory properties [18]. These cells are expanded in vitro through ADORA1and A2A-mediated autocrine signaling and are able to inhibit effector T cell functions through the production of ADO and IL-10 [19].
Myeloid-derived suppressor cells (MDSC) and mesenchymal stem cells (MSC) are populations with regulatory functions, characterized by the presence of adenosinergic ectoenzymes. A recent report indicates that $\mathrm{CD} 11 \mathrm{~b}^{+} \mathrm{CD} 33^{+}$ MDSC in peripheral blood and tumor tissues from nonsmall cell lung cancer (NSCLC) patients express surface ectonucleotidases CD39 and CD73. Such expression is related to the immunosuppressive and chemoprotective effects of MDSC, which favor NSCLC progression [20]. On our side, we showed that MDSC purified from the bone marrow (BM) niche of multiple myeloma patients express CD39 and $\mathrm{CD} 73$, which contribute to the local production of $\mathrm{ADO}$. ADO in the BM is expected to lead to an impairment of the antitumor immune response [21]. The same study pointed out that MSC also express CD38, CD157, and CD203a/PC-1 [21]. Other reports confirmed the presence of CD39 and CD73 in MSC. These cells may lead to the generation of ADO when ATP concentrations are high, such as tissue injuries. However, this is achieved only with the cooperation of activated T lymphocytes, which express high levels of CD39 [22]. On the other hand, other reports demonstrated that cancer-derived MSC express functional CD39 and $\mathrm{CD} 73$, per se able to produce $\mathrm{ADO}$ and hence to suppress $\mathrm{T}$ cell functions [23].

A schematic representation of the expression and function of adenosinergic ectoenzymes on regulatory cell populations is shown in Figure 1.

\section{Autoimmune Diseases}

Autoimmune diseases are chronic conditions initiated by the loss of immunological tolerance to self-antigens and represent a heterogeneous group of disorders that afflict specific target organs or multiple organ systems. The frequent autoimmune diseases include RA (rheumatoid arthritis), SLE (systemic lupus erythematosus), MS (multiple sclerosis), T1DM (type I diabetes mellitus), JIA (juvenile idiopathic arthritis), AIH (autoimmune hepatitis), and so on. The basic function of the human immune system is to distinguish antigens and then eliminate the non-self antigens, to protect the body from infection, tumor, and so on. In normal situations, there is an "alarm" signal which generates various cellular responses that aim to prevent excessive inflammatory response and restore the immune homeostasis. The possible implication of a common point between autoimmune diseases is supported by the presence of common phenotypes between autoimmune disorders: (i) signs and symptoms such as arthralgia, arthritis, alopecia, fatigue, photosensitivity, and Raynaud's phenomenon; (ii) nonspecific autoantibodies (i.e., antinuclear antibodies, rheumatoid factor, and anti-Ro antibodies); (iii) high levels of cytokines (i.e., TNF, IL-1, IL-6, IL-10, IL-17, etc.); and (iv) presence of infiltrating cells as phagocytic macrophages, neutrophils, self-reactive $\mathrm{CD} 4^{+} \mathrm{T}$ helper cells, and self-reactive $\mathrm{CD} 8^{+}$cytolytic $\mathrm{T}$ cells along with smaller numbers of natural killer cells, mast cells, and dendritic cells. Among the T effector cells, Th1, Th17, and Th9 cells contribute to the pathogenesis of autoimmune diseases [24]. In this review, we will point out the implication of $\mathrm{ADO}$ in five autoimmune diseases and/or their 


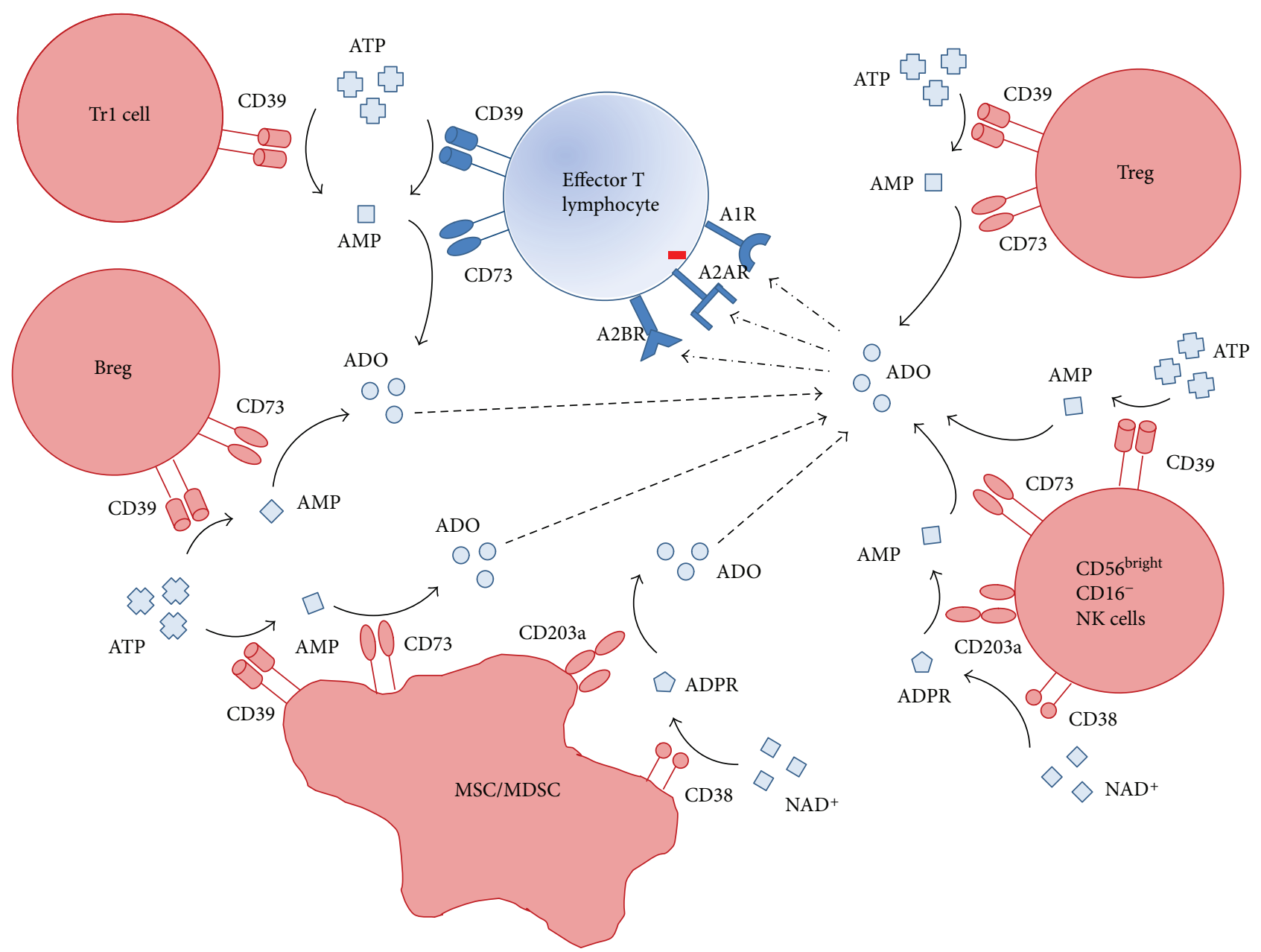

FIGURE 1: Schematic representation of (i) the expression of adenosinergic ectoenzymes on different regulatory cell subsets, (ii) their function in the generation of ADO, and (iii) the inhibitory effect of ADO on immune effector cells.

corresponding animal models: rheumatoid arthritis, multiple sclerosis, juvenile rheumatoid arthritis, autoimmune uveitis, and diabetes mellitus.

Rheumatoid arthritis (RA) is an autoimmune disorder primarily affecting the joints. The underlying mechanism involves a combination of genetic and environmental factors. Several cytokines and their receptors are overexpressed in the synovium, and among them, TNF- $\alpha$ seems to play an important role, and it has been proposed as a therapeutic target [25]. In fact, RA patients treated with the anti-TNF- $\alpha$ antibody (infliximab), alone or in combination with methotrexate (MTX), showed clinical benefits [26].

Multiple sclerosis (MS) is the most famous autoimmune disease attacking the central nervous system. The physiology of MS is monitored by activation of immune-inflammatory, oxidative, and nitrosative stress pathways, related with two main steps: (i) myelin sheath destruction and formation of lesions and (ii) inflammation [27].

Juvenile rheumatoid arthritis is the most common chronic rheumatic disease of childhood and a leading cause of short- and long-term disability. The synovial lymphocyte infiltrate consists mainly of Th1 T cells and, like rheumatoid arthritis, is believed to be a polarization towards Th1 proinflammatory response [28].

Autoimmune uveitis, an inflammatory noninfectious process of the vascular layer of the eye, can lead to visual impairment and, in the absence of a timely diagnosis and suitable therapy, can even result in total blindness. $\mathrm{T}$ cell effector phenotypes and their cytokine pathways seem to be implicated into autoimmune uveitis: T helper 1 (producing interleukin- 2 and interferon- $\gamma$ ) and T helper 2 cells (producing interleukins 4,5 , and 13) exert both pathogenic and protective roles, whereas Thelper 9 (interleukins 9 and 10) and $\mathrm{T}$ helper 17 cells (interleukins 17A, 21, and 22) play exclusively pathogenic roles [29]. Recently, T helper 17 cells have been also implicated in the uveal pathology [30].

Type 1 and 2 diabetes are both caused by immunemediated destruction and dysfunction of pancreatic beta cells, eventually causing defective glucose-stimulated insulin secretion (GSIS) and beta cell apoptosis in diabetes. Recent findings indicate that inflammation and autoimmune recognition of beta cells are triggered by a local production of diabetogenic cytokines, such as IFN- $\gamma$, IL- $1 \beta$, and TNF- $\alpha$, by infiltrated immune cells in pancreatic islets $[31,32]$. 
TABLE 1: Scheme of principal findings regarding the role of ADO and adenosinergic ectoenzymes in autoimmune diseases.

\begin{tabular}{lcr}
\hline Disease & Molecule(s) involved & Principal finding \\
Multiple sclerosis & CD73, CD39 & $\begin{array}{c}\text { Upregulation of CD73 following IFN- } \beta \text { treatment (14, 15) } \\
\text { Loss of regulatory function of CD39 } \text { Tregs }^{(16)}\end{array}$ \\
\hline EAE & CD73, CD39 & $\begin{array}{r}\text { Increase in CD73 expression during disease progression (17) } \\
\text { Resistance to EAE in CD73 KO mice (18, 19) }\end{array}$ \\
\hline CIA & Increased enzymatic activity of CD39 and CD73 in EAE mice (20)
\end{tabular}

\section{Adenosine and Human Autoimmune Diseases}

Several lines of evidence have demonstrated that ADO behaves as the "alarm" signal in vivo. Adenosine activates ADO receptors on target cells, generates multiple cellular responses, and then suppresses the immune response [33]. It has been demonstrated that locally produced ADO can stimulate the release of anti-inflammatory cytokines (i.e., IL-10) and inhibit the release of proinflammatory molecules (i.e., TNF- $\alpha$ and nitric oxide) in animal models of inflammation [34]. Indeed, multiple reports suggest that the onset of autoimmune disorders is-at least in part-related to a partial or complete loss of function of the adenosinergic pathways and to a local defective production of ADO. Table 1 summarizes all these findings.

3.1. Adenosine and Multiple Sclerosis. Notions accumulated in recent years indicate the existence of a link between ADO production and clinical outcome of patients with multiple sclerosis (MS). Airas et al. demonstrated that endothelial cells (ECs) treated with IFN- $\beta$ are able to increase CD73 surface expression in vitro. The same effect was also observed on ECs after systemic treatment of MS patients in vivo with IFN- $\beta$, where an increase in soluble serum CD73 was also observed. Moreover, a correlation between increase in CD73 and clinical outcome of MS patients is reported [35]. In line with this observation, IFN- $\beta$ was shown as increasing the expression of CD73 on blood-brain barrier(BBB-) derived EC and also on astrocytes. This modulation was followed by a reduced transmigration of lymphocytes through BBB-EC. This specificity of action was confirmed by using $\alpha, \beta$-methyleneadenosine- $5^{\prime}$-diphosphate, a specific inhibitor of CD73, which restored the situation quo ante. At the same time, the same group of authors found that the IFN- $\beta$ treatment was followed by increased levels of soluble serum CD73 activity and skin microvascular CD73 expression in the majority of MS patients. This study suggested that ADO might contribute to the beneficial effects of IFN- $\beta$ observed on MS patients [36]. Fletcher et al. demonstrated that CD39, an ectonucleotidase which hydrolyzes ATP, may be also related to MS progression. Indeed, CD39 is expressed on a subset of Tregs. Both $\mathrm{CD} 9^{+}$and $\mathrm{CD} 39^{-}$Treg subsets are able to dampen IFN- $\gamma$ production by effector $\mathrm{T}$ cells, whereas only CD $39^{+}$Tregs are able to suppress IL-17 production by Th17 cells. They found that frequency and suppressive functions of CD39 ${ }^{+}$Tregs are decreased in MS patients and that the inhibition of Th17 cells was related to ADO production through the enzymatic activity of CD39 and CD73 [37].

Other evidences were inferred by the results of studies in the field of experimental autoimmune encephalomyelitis (EAE), an animal model for MS. CD73 expression on Th17 cells appears as increased during disease progression. However, CD73 deficiency did not affect either EAE development or induction of IL-17, IFN- $\gamma$, or GM-CSF, Th17-associated cytokines. Another finding was that CD73 is not required to either promote or limit Th17 cell expansion in vitro [38]. In line with these findings, Millis et al. showed that $\mathrm{cd} 73^{-/-}$ mice are resistant to EAE. However, $\mathrm{CD} 4^{+} \mathrm{T}$ lymphocytes from these mice produced levels of proinflammatory cytokines higher than those observed from wild-type (WT) mice. Furthermore, they may induce EAE when transferred in $\mathrm{cd}^{2} 3^{+/+} \mathrm{T}$ cell-deficient mice. They found that $\mathrm{cd} 73^{-/-}$mice display a lower number of infiltrating lymphocytes in their central nervous system (CNS) than do WT mice. Since they observed a lack of CD73 expression on brain endothelial cells, they concluded that CD73 expression and ADO production are required for the recruitment of lymphocytes into the CNS during EAE development [39]. Other reports showed that such effects were achieved through the induction of CX3CL1 as expressed at the choroid plexus. Indeed, 
CX3CL1 was upregulated in the brain at the acute stage of EAE in WT mice, but not in CD73 KO animals. Moreover, blockade of ADORA2A following EAE induction prevented disease development and the induction of brain CX3CL1 expression. Another finding was that CX3CL1 expression can be upregulated in the brain by antagonizing ADORA2A action. Finally, CX3CL1 blockade protected from EAE development and inhibited lymphocyte entry into the CNS [40]. Hydrolysis of ATP, ADP, and AMP in EAE mice is decreased in blood serum and increased in spinal cord membrane preparations as compared to control mice. A reasonable interpretation is that the function of CD39 and CD73 is regulated during the progression of the disease. An extension of this view is that these molecules represent a target for novel therapeutic strategies in immune-mediated diseases, primarily in MS [41].

Another study demonstrated that ADORA2a was upregulated on $\mathrm{T}$ cells and macrophages/microglia in the CNS of EAE mice. Accordingly, ADORA2a-deficient mice showed an exacerbated disease, with increased frequencies of IFN- $\gamma$-, IL-17-, and GM-CSF-producing $\mathrm{CD}^{+} \mathrm{T}$ cells and higher numbers of inflammatory lesions in the early stage. However, EAE quickly ameliorated and myelin debris accumulation was lower in ADORA2a $\mathrm{a}^{-1-}$ mice. Finally, activation of ADORA2a inhibited myelin phagocytosis by macrophages/ microglia in vitro as well as migration of $\mathrm{T}$ cells, macrophages, and primary microglia [42].

ADORA1 is also involved in the development of EAE. Indeed, it has been demonstrated that $\mathrm{ADORA}^{-1-}$ mice developed a severe progressive-relapsing form of EAE, as compared to WT mice, with worsened demyelination, axonal injury, and enhanced activation of microglia/macrophages. In addition, proinflammatory gene expression was upregulated, whereas anti-inflammatory genes were suppressed [43].

3.2. Adenosine and Rheumatoid Arthritis. Several authors have demonstrated the role of $\mathrm{ADO}$ and adenosinergic ectoenzymes in the context of RA. Flogel et al. demonstrated that the expression of CD73 and ADORA2A is strongly upregulated on neutrophils and inflammatory monocytes isolated from synovial fluids of mice with collagen-induced arthritis (CIA), a well-established mouse model of RA, as compared to controls [44]. Another study was focused on the analysis of the susceptibility of $\mathrm{CD}_{73}^{-1-}$ mice to CIA. CD73-deficient mice are significantly more susceptible to CIA than are wild-type mice. Further, the production of proinflammatory cytokines in the joints, Th1 T cell responses, and joint destruction were all increased. Moreover, a delayed anticollagen IgG response was detected in CD73-deficient mice, thus suggesting a defective isotype class switching. The authors have demonstrated that CD73 expression on nonhematopoietic cells was important for protecting from CIA development [45]. CD39 ${ }^{+}$Tregs have been characterized also in the context of RA. Indeed, it has been demonstrated that the frequency of this cell subset is increased in the peripheral blood $(\mathrm{PB})$ of RA patients and that these cells are also abundant in the synovial fluid (SF). Also in the synovium, CD39 ${ }^{+}$Tregs are able to suppress different effector $\mathrm{T}$ cell functions, including production of IFN- $\gamma$, TNF, and
IL-17F, but they do not limit IL-17A secretion, as previously reported [46]. The role of $\mathrm{CD} 9^{+}$Tregs as key factors as immune modulators in the context of RA has been demonstrated by Peres et al., who have analyzed the frequency of this cell subset in the PB of RA patients treated for three months with MTX. They found that such frequency was increased in RA patients that were responsive to MTX, but not in unresponsive patients, as compared to healthy controls. Furthermore, they demonstrated that Tregs from unresponsive patients express lower levels of CD39, produce less amounts of ADO (starting from ATP), and display a decreased regulatory activity, as compared to Tregs from MTX-responsive patients. In a prospective study, they demonstrated that unresponsive patients displayed a lower expression of CD39 on Tregs than did responsive patients and healthy controls. Moreover, CD39 blockade dampened the antiarthritic effect of MTX treatment in a murine model of RA, thus suggesting that CD39 and ADO production is involved in the mechanism of action of MTX and is required for the responsiveness to the treatment. The authors concluded that low expression of CD39 on Tregs could represent a biomarker for identifying MTXresistant RA patients [47].

3.3. Adenosine and Juvenile Idiopathic Arthritis. We have demonstrated an altered function of adenosinergic ectoenzymes in the context of juvenile idiopathic arthritis (JIA), a pediatric form of RA. In particular, the expression and function of CD38 and CD73 were decreased in CD16 ${ }^{-}$CD $56^{\text {bright }}$ NK cells isolated from synovial fluid of JIA patients. These cells displayed an altered kinetic for ADO production and failed to inhibit autologous $\mathrm{CD}^{+} \mathrm{T}$ cell proliferation, as compared to those isolated from healthy controls [16]. Other authors have demonstrated that, in JIA patients, the expression of CD73 on $\mathrm{CD}_{19}{ }^{+}$and $\mathrm{CD} 8^{+}$lymphocytes was lower in cells isolated from SF than in those from PB. Consequently, SF $\mathrm{CD}^{+}$cells produced less amounts of ADO than did PB counterparts. Finally, they found that CD73 expression in SF cells was lower in patients with an extended oligoarticular form than in those with milder forms, thus suggesting that CD73 expression and ADO production correlated with disease severity, due to a low anti-inflammatory activity [48].

3.4. Adenosine and Autoimmune Uveitis. A putative role of $\mathrm{ADO}$ has been also described in experimental autoimmune uveitis (EAU), a murine model of human autoimmune uveitis. It has been demonstrated that the recovery from EAU is mediated by suppressor antigen-presenting cells, which present autoantigens and produce ADO to activate antigen-specific Tregs, through ADORA2A. These inducible Tregs can, in turn, suppress the autoimmune disease through a PD-1/PD-L1-dependent mechanism [49]. It has been also demonstrated that mesenchymal stem cells (MSC) are able to attenuate the severity of the disease. Such therapeutic effect was reverted by pretreating MSC with a CD73 inhibitor, thus suggesting that this immune-modulatory activity was related to $\mathrm{ADO}$, which is produced through the cooperation between CD73 and CD39 and in turn abrogates T cell functions [50]. 
$\gamma \delta \mathrm{T}$ cells can either enhance or inhibit an adaptive immune response and are important in the context of EAU. It has been reported that $\gamma \delta \mathrm{T}$ cells expressed CD73 during the different stages of EAU, and a low CD73 expression on $\gamma \delta \mathrm{T}$ cells correlated with enhanced Th17 response. The expression of CD73 on $\gamma \delta \mathrm{T}$ cells is transiently downregulated upon activation. Moreover, $\gamma \delta \mathrm{T}$ cells isolated from $\mathrm{CD}_{73}^{-l-}$ mice are more potent at inducing a Th-17 response and eye pathology in $\gamma \delta^{-/-}$-recipient mice than are $\gamma \delta \mathrm{T}$ cells isolated from WT mice. On the other hand, $\gamma \delta \mathrm{T}$ cells isolated from WT mice, but not those isolated from $\mathrm{CD}_{73^{-/-}}$mice, are able to inhibit $\mathrm{T}$ cell proliferation in vitro. Collectively, these data suggest that targeting CD73 expression on $\gamma \delta \mathrm{T}$ cells might control both their pro- or anti-inflammatory effects [51].

3.5. Adenosine and Diabetes. A possible role of adenosinergic ectoenzymes and ADO has been recently hypothesized in a multiple low-dose streptozotocin- (MLDS-) induced diabetes, a murine model of human T1DM. In particular, the authors investigated the role of CD39, using wild-type mice, CD39 KO mice, and mice overexpressing CD39 that were subsequently subjected to MLDS. They demonstrated that CD39KO mice developed diabetes more rapidly and with higher frequency than did WT mice. In contrast, overexpression of CD39 protected mice from diabetes, through the production of $\mathrm{ADO}$ and activation of ADORA2A and ADORA2B [52]. In this line, it has been previously demonstrated that mice lacking ADORA2A (A2AR KO) are highly susceptible to MLDS-induced diabetes, with the presence of hyperproliferative $\mathrm{T}$ cells [53]. In contrast, CD73 $\mathrm{KO}$ mice were protected from MLDS-induced diabetes as compared to wild-type mice [54].

\section{Adenosine in Other Inflammatory Diseases}

Inflammatory diseases include autoimmune diseases and a vast array of disorders and conditions that are characterized by inflammation. This includes allergy, asthma, celiac disease, glomerulonephritis and other renal diseases, hepatitis, inflammatory bowel disease, preperfusion injury, and inflammatory diseases related to transplant. We have here investigated the role of $\mathrm{ADO}$ in the context of skin allergy, renal inflammatory diseases, and graft-versus-host disease (GvHD).

4.1. CD73 in Inflamed Human Skin. CD73 is highly expressed on endothelial cells and plays a role in mediating the adhesion of leukocytes to endothelial cells. Consequently, CD73 regulates the entry of the latter cells in the vascular network and, in turn, their recruitment to the site of inflammation. Moreover, $\mathrm{ADO}$ produced by $\mathrm{CD}^{+} 3^{+}$endothelial cells exerts anti-inflammatory effects, helping to maintain the endothelial barrier function [55-57]. Arvilommi et al. have investigated the role of CD73 in the migration of activated lymphocytes to inflamed skin, in the context of idiopathic and allergic disorders of the skin. They demonstrated that CD73 is also expressed on activated lymphocytes, and skinhoming lymphocytes mostly expressed both CD73 and cutaneous lymphocyte antigen (CLA). Moreover, the treatment of peripheral blood lymphocytes with an anti-CD73 monoclonal antibody significantly reduced the binding of the latter cells to the vascular endothelium of the inflamed skin, thus suggesting the role of CD73 in the recruitment of activated lymphocytes in this context [58].

4.2. CD73 in Renal Diseases. The important role of CD73 on endothelial cells in the control of leukocyte trafficking has been investigated also in mice with renal injury. In fact, $\mathrm{CD}^{-1-}$ mice showed autoimmune inflammation with glomerulitis and peritubular capillaritis, glomerular deposition of immunoglobulins, and complement and enhanced presence of lymphocytes and macrophages in the interstitium. Moreover, vascular inflammation was associated with enhanced serum levels of proinflammatory cytokines and chemokines. Finally, a reduced number of podocytes and endothelial fenestrations were observed in $\mathrm{CD} 73^{-1-}$ mice. These data demonstrated that a lack of CD73 reduced renal function and is associated with autoimmune inflammation [59].

4.3. Adenosine and Graft-versus-Host Disease. MSC exert their regulatory activity through the release of soluble immunosuppressive molecules, such as TGF- $\beta 1, \mathrm{HGF}, \mathrm{IDO}, \mathrm{PGE}_{2}$, IL-10, and HLA-G [60]. For their immunosuppressive potential, MSC have been proposed as a therapeutic tool in the treatment of severe acute GvHD. Recently, Sangiorgi et al. have demonstrated that, upon stimulation with a tolllike receptor 9 agonist, bone marrow- (BM-) derived MSC (i) upregulated CD73 and CD39 expression on their surface and (ii) showed increased immunosuppressive functions. Thus, they conclude that ADO, generated by the CD39/ CD73 pathway, may also play a role in the immunomodulatory function of MSC [61].

Like MSC, human-derived gingival mesenchymal stem cells (GMSCs) are able to suppress immune responses and $\mathrm{T}$ cell-mediated CIA in animals. It has been demonstrated that GMSCs are able to suppress the proliferation of PBMC and $\mathrm{T}$ cells in vitro. When cotransferred with PBMC in a murine model of GvDH, GMSCs are able to suppress the engraftment of human cells and to prolong the survival of mice. It has been demonstrated that the mechanism of GMSC-mediated immunosuppression involves the enzymatic function of CD39 and CD73 and production of ADO. These cells represent a potential clinical option for the treatment of GvHD and autoimmune diseases [62].

The role of CD73 and ADO in the control of GvHD was also supported by the finding that Tregs from CD73 KO mice are unable to mitigate GvHD mortality, compared to WT Tregs. Moreover, blockade of the ADO A2a receptor exacerbated GvHD, thus suggesting that ADO is involved in this process, through the interaction with the $\mathrm{A} 2 \mathrm{a}$ receptor. Finally, blockade of CD73 induced the expansion of alloreactive $\mathrm{T}$ cells, which exacerbated GvHD and enhanced the graft-versus-tumor effect. Collectively, these data demonstrated that blocking CD73 activity might be relevant in the context of BM transplantation $[63,64]$. 


\section{Therapeutic Implications for Autoimmune Disorders}

Several studies support the concept that ADO, as well as enzymes belonging to adenosinergic pathways for ADO production, might represent promising therapeutic targets for autoimmune and inflammatory diseases.

Flogel et al. analyzed the effects of 2-(cyclohexyl methylthio) adenosine $5^{\prime}$-monophosphate (chet-AMP), a phosphorylated ADORA2A agonist (prodrug) that requires CD73 activity to become activated, in mice with CIA. The administration of chet-AMP triggers immunosuppressive and anti-inflammatory activities, through a CD73-mediated conversion of chet-AMP to functional chet-ADO. These findings suggested that ADOR agonists may represent a novel therapeutic target for patients with RA [44]. Similar data were obtained in another study, where the administration of an ADORA2A agonist to $\mathrm{CD} 73^{-/-}$mice resulted in arthritis incidence similar to wild-type mice, thus confirming the protective role for A2A signaling in the context of CIA [45]. Similar results have been obtained using agonists of ADORA3: the mechanism underlying this beneficial anti-inflammatory effect was related to the suppression of TNF- $\alpha$ production $[65,66]$.

Ingwersen et al. described a dual role for ADORA2a in the context of EAE. Indeed, preventive treatment with the ADORA2a-specific agonist ameliorated disease, whereas treatment with the same agonist after disease onset exacerbated disease progression and tissue destruction. These data suggested that ADORA2a provides anti-inflammatory effects on T cells and protection at early stages, whereas in the later stages the activation of this receptor may sustain tissue damage within the inflamed CNS [42]. Liu et al. observed a limitation of disease progression, driven by a reduced $\mathrm{T}$ cell proliferation, CNS infiltration, and cytokine production, in EAE mice treated with an ADORA2a agonist [67]. Tsutsui et al. demonstrated that treatment of EAE mice with caffeine increased the expression of ADORA1 on microglia, with a following reduction of EAE severity. Such effect was further enhanced by a concomitant treatment with the ADORA1 agonist, thus suggesting that ADORA1 represents a therapeutic target to modulate neuroinflammation in MS and other demyelinating diseases [43].

A therapeutic activity of $\mathrm{ADO}$ receptor agonists was also observed in the context of other preclinical models of autoimmune diseases. In diabetic mice, a nonselective ADO receptor agonist prevented diabetes development in mice treated with MLDS or cyclophosphamide. Such effect was reverted using ADORA2b but not ADORA1 or ADORA2a antagonists, thus suggesting that only ADORA2b represents a promising therapeutic target for type 1 diabetes [68]. ADORA2a represents a promising therapeutic target for myasthenia gravis. Indeed, it has been demonstrated in rats with experimental autoimmune myasthenia gravis (EAMG) that the stimulation of ADORA2a with a specific agonist reduced the proliferation of acetylcholine receptor- (AChR-) specific autoreactive lymphocytes and the production of anti-AChR antibodies and ameliorated disease severity [69].
In mice with EAU, treatment with CF-101, an agonist of ADORA3, ameliorated pathological manifestation of the disease, through the inhibition of autoreactive $\mathrm{T}$ cell proliferation and release of proinflammatory cytokines. This study pointed out a role for ADORA3 as a therapeutic target for human uveitis [70]. In contrast, ADORA2b activation using a specific agonist enhanced the development of the disease in the same preclinical model, through the activation of Th-17 response mediated by dendritic cells [71].

Limited clinical trials are ongoing targeting $\mathrm{ADO}, \mathrm{ADO}$ receptors, and other related molecules in patients with autoimmune/inflammatory diseases. CF-101 has been tested in phase I, phase IIa, and phase IIb human studies. The treatment was safe and well tolerated and induced an antiinflammatory and antirheumatic effect in RA patients. Moreover, a significant correlation between ADORA3 expression level and response to drug was reported, thus suggesting that ADORA3 may represent a biologic marker to predict a response to the drug in patients $[66,72,73]$.

A phase I/II randomized study was carried out on patients who underwent hematopoietic stem cell transplantation treated with pentostatin, a specific inhibitor of ADA. The results demonstrated that GvHD rates are significantly decreased in patients treated with pentostatin associated with the standard treatment. Accordingly, transplant success rates were higher in treated patients than in controls [74]. A phase II study on HSC-transplanted patients demonstrated a response rate to pentostatin (administered with corticosteroids) of $55 \%$ and a survival rate of $78 \%$ at $1-2$ years [75]. Similarly, a phase II study in children demonstrated a response rate to pentostatin of $53 \%$ and a survival rate of $69 \%$ at 3 years [76]. In contrast, another phase II trial demonstrated a complete response to pentostatin (administered with corticosteroids) only in $38 \%$ of patients and a 9 -month overall survival of $47 \%$, with an incidence of severe infections of $57 \%$ [77].

\section{Conclusions}

In the last years, several authors reported that extracellular $\mathrm{ADO}$, produced by ectoenzymes belonging to the "classical" adenosinergic pathway (CD39 and CD73), might be involved in the control of the immune response in the context of human autoimmune/inflammatory diseases. Indeed, alterations of the expression and function of both CD39 and CD73 have been reported to be related to the onset of these diseases. On the contrary, overexpression of these molecules, as well as agonists of ADO receptors, is able to mitigate the immune responses and the inflammatory reaction that are crucial for the development of the disease. So far, little is known regarding the expression and function of ectoenzymes that form the "alternative" pathway for ADO production (CD38, CD203a/PC-1, and CD73). We have demonstrated that CD203a/PC-1 is specifically expressed by $\mathrm{CD} 16^{-} \mathrm{CD} 56^{\text {bright }} \mathrm{NK}$ cells (that exert regulatory activities) and not by cytotoxic $\mathrm{CD} 16^{+} \mathrm{CD} 56^{\mathrm{dim}} \mathrm{NK}$ cells. Moreover, we have demonstrated that the regulatory activity of the former cells is altered in JIA patients, and such alteration was related to a lower expression of CD38 and CD73, 
thus suggesting that also the "alternative" pathway for ADO production might be altered in autoimmune/inflammatory diseases. In the future, it would be interesting to characterize the expression and function of CD38 and CD203a/PC-1 in other human autoimmune diseases. Indeed, these molecules, as well as CD39 and CD73, might represent a promising target for novel and effective therapeutic approaches.

\section{Conflicts of Interest}

The authors declare that they have no conflicts of interest.

\section{References}

[1] B. B. Fredholm, A. P. IJzerman, K. A. Jacobson, K. N. Klotz, and J. Linden, "International Union of Pharmacology. XXV. Nomenclature and classification of adenosine receptors," Pharmacological Reviews, vol. 63, no. 1, pp. 1-34, 2001.

[2] L. Antonioli, C. Blandizzi, P. Pacher, and G. Hasko, "Immunity, inflammation and cancer: a leading role for adenosine," Nature Reviews Cancer, vol. 13, no. 12, pp. 842-857, 2013.

[3] Z. H. Nemeth, S. J. Leibovich, E. A. Deitch et al., "Adenosine stimulates CREB activation in macrophages via a p38 MAPK-mediated mechanism," Biochemical and Biophysical Research Communications, vol. 312, no. 4, pp. 883-888, 2003.

[4] D. W. Hoskin, J. S. Mader, S. J. Furlong, D. M. Conrad, and J. Blay, "Inhibition of $\mathrm{T}$ cell and natural killer cell function by adenosine and its contribution to immune evasion by tumor cells (review)," International Journal of Oncology, vol. 32, no. 3, pp. 527-535, 2008.

[5] A. Ben Addi, A. Lefort, X. Hua et al., "Modulation of murine dendritic cell function by adenine nucleotides and adenosine: involvement of the $\mathrm{A}_{2 \mathrm{~B}}$ receptor," European Journal of Immunology, vol. 38, no. 6, pp. 1610-1620, 2008.

[6] V. Le Vraux, Y. L. Chen, I. Masson et al., "Inhibition of human monocyte TNF production by adenosine receptor agonists," Life Sciences, vol. 52, no. 24, pp. 1917-1924, 1993.

[7] L. Antonioli, P. Pacher, E. S. Vizi, and G. Hasko, "CD39 and CD73 in immunity and inflammation," Trends in Molecular Medicine, vol. 19, no. 6, pp. 355-367, 2013.

[8] B. Allard, M. S. Longhi, S. C. Robson, and J. Stagg, "The ectonucleotidases CD39 and CD73: novel checkpoint inhibitor targets," Immunological Reviews, vol. 276, no. 1, pp. 121144, 2017.

[9] G. Fedele, I. Sanseverino, K. D'Agostino et al., "Unconventional, adenosine-producing suppressor $\mathrm{T}$ cells induced by dendritic cells exposed to BPZE1 pertussis vaccine," Journal of Leukocyte Biology, vol. 98, no. 4, pp. 631-639, 2015.

[10] A. L. Horenstein, A. Chillemi, G. Zaccarello et al., "A CD38/ CD203a/CD73 ectoenzymatic pathway independent of CD39 drives a novel adenosinergic loop in human T lymphocytes," Oncoimmunology, vol. 2, no. 9, article e26246, 2013.

[11] J. B. Rose and I. R. Coe, "Physiology of nucleoside transporters: back to the future," Physiology, vol. 23, pp. 41-48, 2008.

[12] G. Cristalli, S. Costanzi, C. Lambertucci et al., "Adenosine deaminase: functional implications and different classes of inhibitors," Medicinal Research Reviews, vol. 21, no. 2, pp. 105-128, 2001.

[13] W. P. Schrader, B. Pollara, and H. J. Meuwissen, "Characterization of the residual adenosine deaminating activity in the spleen of a patient with combined immunodeficiency disease and adenosine deaminase deficiency," Proceedings of the National Academy of Sciences of the United States of America, vol. 75, no. 1, pp. 446-450, 1978.

[14] A. A. Welihinda, M. Kaur, K. S. Raveendran, and E. P. Amento, "Enhancement of inosine-mediated $\mathrm{A}_{2 \mathrm{~A}} \mathrm{R}$ signaling through positive allosteric modulation," Cellular Signalling, vol. 42, pp. 227-235, 2018.

[15] M. Mandapathil, B. Hilldorfer, M. J. Szczepanski et al., "Generation and accumulation of immunosuppressive adenosine by human $\mathrm{CD} 4{ }^{+} \mathrm{CD} 25^{\text {high }} \mathrm{FOXP} 3^{+}$regulatory T cells," The Journal of Biological Chemistry, vol. 285, no. 10, pp. 71767186, 2010.

[16] F. Morandi, A. L. Horenstein, A. Chillemi et al., "CD56 ${ }^{\text {bright }} \mathrm{CD} 16^{-} \mathrm{NK}$ cells produce adenosine through a CD38-mediated pathway and act as regulatory cells inhibiting autologous $\mathrm{CD}^{+} \mathrm{T}$ cell proliferation," The Journal of Immunology, vol. 195, no. 3, pp. 965-972, 2015.

[17] I. D. Mascanfroni, M. C. Takenaka, A. Yeste et al., "Metabolic control of type 1 regulatory T cell differentiation by AHR and HIF1- $\alpha$," Nature Medicine, vol. 21, no. 6, pp. 638-646, 2015.

[18] F. Figueiró, L. Muller, S. Funk, E. K. Jackson, A. M. Battastini, and T. L. Whiteside, "Phenotypic and functional characteristics of CD39 $9^{\text {high }}$ human regulatory B cells (Breg)," Oncoimmunology, vol. 5, no. 2, article e1082703, 2016.

[19] Z. Saze, P. J. Schuler, C. S. Hong, D. Cheng, E. K. Jackson, and T. L. Whiteside, "Adenosine production by human B cells and B cell-mediated suppression of activated T cells," Blood, vol. 122, no. 1, pp. 9-18, 2013.

[20] J. Li, L. Wang, X. Chen et al., "CD39/CD73 upregulation on myeloid-derived suppressor cells via TGF- $\beta$-mTOR-HIF- 1 signaling in patients with non-small cell lung cancer," Oncoimmunology, vol. 6, no. 6, article e1320011, 2017.

[21] V. Quarona, V. Ferri, A. Chillemi et al., "Unraveling the contribution of ectoenzymes to myeloma life and survival in the bone marrow niche," Annals of the New York Academy of Sciences, vol. 1335, no. 1, pp. 10-22, 2015.

[22] E. Kerkela, A. Laitinen, J. Rabina et al., “Adenosinergic immunosuppression by human mesenchymal stromal cells requires co-operation with T cells," Stem Cells, vol. 34, no. 3, pp. 781790, 2016.

[23] M. de Lourdes Mora-Garcia, R. Garcia-Rocha, O. MoralesRamirez et al., "Mesenchymal stromal cells derived from cervical cancer produce high amounts of adenosine to suppress cytotoxic T lymphocyte functions," Journal of Translational Medicine, vol. 14, no. 1, p. 302, 2016.

[24] A. Jager and V. K. Kuchroo, "Effector and regulatory T-cell subsets in autoimmunity and tissue inflammation," Scandinavian Journal of Immunology, vol. 72, no. 3, pp. 173184, 2010.

[25] M. Feldmann and S. R. N. Maini, "Role of cytokines in rheumatoid arthritis: an education in pathophysiology and therapeutics," Immunological Reviews, vol. 223, no. 1, pp. 719, 2008.

[26] F. C. Breedveld, P. Emery, E. Keystone et al., "Infliximab in active early rheumatoid arthritis," Annals of the Rheumatic Diseases, vol. 63, no. 2, pp. 149-155, 2004.

[27] A. J. Thompson, B. L. Banwell, F. Barkhof et al., "Diagnosis of multiple sclerosis: 2017 revisions of the McDonald criteria," The Lancet Neurology, vol. 17, no. 2, pp. 162-173, 2018.

[28] S. Grevich and S. Shenoi, "Update on the management of systemic juvenile idiopathic arthritis and role of IL-1 and IL-6 
inhibition," Adolescent Health, Medicine and Therapeutics, vol. 8, pp. 125-135, 2017.

[29] R. Horai and R. R. Caspi, "Cytokines in autoimmune uveitis," Journal of Interferon \& Cytokine Research, vol. 31, no. 10, pp. 733-744, 2011.

[30] T. Qin, "Upregulation of DR3 expression in $\mathrm{CD}^{+} \mathrm{T}$ cells promotes secretion of IL-17 in experimental autoimmune uveitis," Molecular Vision, vol. 17, pp. 3486-3493, 2011.

[31] M. Cnop, N. Welsh, J. C. Jonas, A. Jorns, S. Lenzen, and D. L. Eizirik, "Mechanisms of pancreatic $\beta$-cell death in type 1 and type 2 diabetes: many differences, few similarities," Diabetes, vol. 54, Supplement 2, pp. S97-S107, 2005.

[32] Y. Imai, A. D. Dobrian, M. A. Morris, and J. L. Nadler, "Islet inflammation: a unifying target for diabetes treatment?" Trends in Endocrinology \& Metabolism, vol. 24, no. 7, pp. 351-360, 2013.

[33] M. M. Faas, T. Saez, and P. de Vos, "Extracellular ATP and adenosine: the Yin and Yang in immune responses?," Molecular Aspects of Medicine, vol. 55, pp. 9-19, 2017.

[34] G. Hasko, C. Szabo, Z. H. Nemeth, V. Kvetan, S. M. Pastores, and E. S. Vizi, "Adenosine receptor agonists differentially regulate IL-10, TNF- $\alpha$, and nitric oxide production in RAW 264.7 macrophages and in endotoxemic mice," The Journal of Immunology, vol. 157, no. 10, pp. 4634-4640, 1996.

[35] L. Airas, J. Niemela, G. Yegutkin, and S. Jalkanen, "Mechanism of action of IFN- $\beta$ in the treatment of multiple sclerosis: a special reference to CD73 and adenosine," Annals of the New York Academy of Sciences, vol. 1110, no. 1, pp. 641-648, 2007.

[36] J. Niemela, I. Ifergan, G. G. Yegutkin, S. Jalkanen, A. Prat, and L. Airas, "IFN- $\beta$ regulates CD73 and adenosine expression at the blood-brain barrier," European Journal of Immunology, vol. 38, no. 10, pp. 2718-2726, 2008.

[37] J. M. Fletcher, R. Lonergan, L. Costelloe et al., "CD39 $9^{+}$Foxp3 ${ }^{+}$ regulatory $\mathrm{T}$ cells suppress pathogenic Th17 cells and are impaired in multiple sclerosis," The Journal of Immunology, vol. 183, no. 11, pp. 7602-7610, 2009.

[38] G. Hernandez-Mir and M. J. McGeachy, "CD73 is expressed by inflammatory Th17 cells in experimental autoimmune encephalomyelitis but does not limit differentiation or pathogenesis," PLoS One, vol. 12, no. 3, article e0173655, 2017.

[39] J. H. Mills, L. F. Thompson, C. Mueller et al., "CD73 is required for efficient entry of lymphocytes into the central nervous system during experimental autoimmune encephalomyelitis," Proceedings of the National Academy of Sciences of the United States of America, vol. 105, no. 27, pp. 93259330, 2008.

[40] J. H. Mills, L. M. Alabanza, D. A. Mahamed, and M. S. Bynoe, "Extracellular adenosine signaling induces CX3CL1 expression in the brain to promote experimental autoimmune encephalomyelitis," Journal of Neuroinflammation, vol. 9, no. 1, p. 193, 2012.

[41] I. Lavrnja, I. Bjelobaba, M. Stojiljkovic et al., "Time-course changes in ectonucleotidase activities during experimental autoimmune encephalomyelitis," Neurochemistry International, vol. 55, no. 4, pp. 193-198, 2009.

[42] J. Ingwersen, B. Wingerath, J. Graf et al., "Dual roles of the adenosine A2a receptor in autoimmune neuroinflammation," Journal of Neuroinflammation, vol. 13, no. 1, p. 48, 2016.

[43] S. Tsutsui, J. Schnermann, F. Noorbakhsh et al., "A1 adenosine receptor upregulation and activation attenuates neuroinflammation and demyelination in a model of multiple sclerosis,"
The Journal of Neuroscience, vol. 24, no. 6, pp. 15211529, 2004.

[44] U. Flogel, S. Burghoff, P. L. E. M. van Lent et al., "Selective activation of adenosine $A_{2 \mathrm{~A}}$ receptors on immune cells by a CD73-dependent prodrug suppresses joint inflammation in experimental rheumatoid arthritis," Science Translational Medicine, vol. 4, no. 146, article 146ra108, 2012.

[45] P. Chrobak, R. Charlebois, P. Rejtar, R. El Bikai, B. Allard, and J. Stagg, "CD73 plays a protective role in collagen-induced arthritis," The Journal of Immunology, vol. 194, no. 6, pp. 2487-2492, 2015.

[46] J. Herrath, K. Chemin, I. Albrecht, A. I. Catrina, and V. Malmstrom, "Surface expression of CD39 identifies an enriched Treg-cell subset in the rheumatic joint, which does not suppress IL-17A secretion," European Journal of Immunology, vol. 44, no. 10, pp. 2979-2989, 2014.

[47] R. S. Peres, F. Y. Liew, J. Talbot et al., "Low expression of CD39 on regulatory $\mathrm{T}$ cells as a biomarker for resistance to methotrexate therapy in rheumatoid arthritis," Proceedings of the National Academy of Sciences of the United States of America, vol. 112, no. 8, pp. 2509-2514, 2015.

[48] S. Botta Gordon-Smith, S. Ursu, S. Eaton, H. Moncrieffe, and L. R. Wedderburn, "Correlation of low CD73 expression on synovial lymphocytes with reduced adenosine generation and higher disease severity in juvenile idiopathic arthritis," Arthritis \& Rhematology, vol. 67, no. 2, pp. 545-554, 2015.

[49] D. J. Lee and A. W. Taylor, "Recovery from experimental autoimmune uveitis promotes induction of antiuveitic inducible Tregs," Journal of Leukocyte Biology, vol. 97, no. 6, pp. 1101-1109, 2015.

[50] X. Chen, H. Shao, Y. Zhi et al., "CD73 pathway contributes to the immunosuppressive ability of mesenchymal stem cells in intraocular autoimmune responses," Stem Cells and Development, vol. 25, no. 4, pp. 337-346, 2016.

[51] D. Liang, A. Zuo, R. Zhao et al., "CD73 expressed on $\gamma \delta$ T cells shapes their regulatory effect in experimental autoimmune uveitis," PLoS One, vol. 11, no. 2, article e0150078, 2016.

[52] J. S. J. Chia, J. L. McRae, H. E. Thomas et al., "The protective effects of CD39 overexpression in multiple low-dose streptozotocin-induced diabetes in mice," Diabetes, vol. 62, no. 6, pp. 2026-2035, 2013.

[53] S. Deaglio, K. M. Dwyer, W. Gao et al., "Adenosine generation catalyzed by CD39 and CD73 expressed on regulatory T cells mediates immune suppression," Journal of Experimental Medicine, vol. 204, no. 6, pp. 1257-1265, 2007.

[54] J. S. Chia, J. L. McRae, P. J. Cowan, and K. M. Dwyer, "The CD39-adenosinergic axis in the pathogenesis of immune and nonimmune diabetes," Journal of Biomedicine and Biotechnology, vol. 2012, Article ID 320495, 7 pages, 2012.

[55] G. Bellingan, M. Maksimow, D. C. Howell et al., "The effect of intravenous interferon-beta-1a (FP-1201) on lung CD73 expression and on acute respiratory distress syndrome mortality: an open-label study," The Lancet Respiratory Medicine, vol. 2, no. 2, pp. 98-107, 2014.

[56] J. Reutershan, I. Vollmer, S. Stark, R. Wagner, K. C. Ngamsri, and H. K. Eltzschig, "Adenosine and inflammation: CD39 and CD73 are critical mediators in LPS-induced PMN trafficking into the lungs," The FASEB Journal, vol. 23, no. 2, pp. 473482, 2009.

[57] P. F. Lennon, C. T. Taylor, G. L. Stahl, and S. P. Colgan, "Neutrophil-derived 5 '-adenosine monophosphate promotes 
endothelial barrier function via CD73-mediated conversion to adenosine and endothelial $\mathrm{A}_{2 \mathrm{~B}}$ receptor activation," The Journal of Experimental Medicine, vol. 188, no. 8, pp. 14331443, 1998.

[58] A. M. Arvilommi, M. Salmi, L. Airas, K. Kalimo, and S. Jalkanen, "CD73 mediates lymphocyte binding to vascular endothelium in inflamed human skin," European Journal of Immunology, vol. 27, no. 1, pp. 248-254, 1997.

[59] C. Blume, A. Felix, N. Shushakova et al., "Autoimmunity in CD73/Ecto- $5^{\prime}$-nucleotidase deficient mice induces renal injury," PLoS One, vol. 7, no. 5, article e37100, 2012.

[60] E. Soleymaninejadian, K. Pramanik, and E. Samadian, "Immunomodulatory properties of mesenchymal stem cells: cytokines and factors," American Journal of Reproductive Immunology, vol. 67, no. 1, pp. 1-8, 2012.

[61] B. Sangiorgi, H. T. De Freitas, J. L. Schiavinato et al., "DSP30 enhances the immunosuppressive properties of mesenchymal stromal cells and protects their suppressive potential from lipopolysaccharide effects: a potential role of adenosine," Cytotherapy, vol. 18, no. 7, pp. 846-859, 2016.

[62] F. Huang, M. Chen, W. Chen et al., "Human gingiva-derived mesenchymal stem cells inhibit xeno-graft-versus-host disease via CD39-CD73-adenosine and IDO signals," Frontiers in Immunology, vol. 8, p. 68, 2017.

[63] L. Wang, J. Fan, S. Chen, Y. Zhang, T. J. Curiel, and B. Zhang, "Graft-versus-host disease is enhanced by selective CD73 blockade in mice," PLoS One, vol. 8, no. 3, article e58397, 2013.

[64] H. Tsukamoto, P. Chernogorova, K. Ayata et al., "Deficiency of CD73/ecto- $5^{\prime}$-nucleotidase in mice enhances acute graftversus-host disease," Blood, vol. 119, no. 19, pp. 45544564, 2012.

[65] E. Baharav, S. Bar-Yehuda, L. Madi et al., "Antiinflammatory effect of $\mathrm{A}_{3}$ adenosine receptor agonists in murine autoimmune arthritis models," The Journal of Rheumatology, vol. 32, no. 3, pp. 469-476, 2005.

[66] S. Bar-Yehuda, M. H. Silverman, W. D. Kerns, A. Ochaion, S. Cohen, and P. Fishman, "The anti-inflammatory effect of $\mathrm{A}_{3}$ adenosine receptor agonists: a novel targeted therapy for rheumatoid arthritis," Expert Opinion on Investigational Drugs, vol. 16, no. 10, pp. 1601-1613, 2007.

[67] Y. Liu, H. Zou, P. Zhao et al., "Activation of the adenosine A2A receptor attenuates experimental autoimmune encephalomyelitis and is associated with increased intracellular calcium levels," Neuroscience, vol. 330, pp. 150-161, 2016.

[68] Z. H. Nemeth, D. Bleich, B. Csoka et al., "Adenosine receptor activation ameliorates type 1 diabetes," The FASEB Journal, vol. 21, no. 10, pp. 2379-2388, 2007.

[69] N. Li, L. Mu, J. Wang et al., "Activation of the adenosine A2A receptor attenuates experimental autoimmune myasthenia gravis severity," European Journal of Immunology, vol. 42, no. 5, pp. 1140-1151, 2012.

[70] S. Bar-Yehuda, D. Luger, A. Ochaion et al., "Inhibition of experimental auto-immune uveitis by the A3 adenosine receptor agonist CF101," International Journal of Molecular Medicine, vol. 28, no. 5, pp. 727-731, 2011.

[71] M. Chen, D. Liang, A. Zuo, H. Shao, H. J. Kaplan, and D. Sun, "An A2B adenosine receptor agonist promotes Th17 autoimmune responses in experimental autoimmune uveitis (EAU) via dendritic cell activation," PLoS One, vol. 10, no. 7, article e0132348, 2015.
[72] P. Fishman and S. Cohen, "The $A_{3}$ adenosine receptor $\left(A_{3} A R\right)$ : therapeutic target and predictive biological marker in rheumatoid arthritis," Clinical Rheumatology, vol. 35, no. 9, pp. 23592362, 2016.

[73] M. H. Silverman, V. Strand, D. Markovits et al., "Clinical evidence for utilization of the $\mathrm{A}_{3}$ adenosine receptor as a target to treat rheumatoid arthritis: data from a phase II clinical trial," The Journal of Rheumatology, vol. 35, no. 1, pp. 4148, 2008.

[74] S. Parmar, B. S. Andersson, D. Couriel et al., "Prophylaxis of graft-versus-host disease in unrelated donor transplantation with pentostatin, tacrolimus, and mini-methotrexate: a phase I/II controlled, adaptively randomized study," Journal of Clinical Oncology, vol. 29, no. 3, pp. 294-302, 2011.

[75] D. A. Jacobsohn, A. R. Chen, M. Zahurak et al., "Phase II study of pentostatin in patients with corticosteroid-refractory chronic graft-versus-host disease," Journal of Clinical Oncology, vol. 25, no. 27, pp. 4255-4261, 2007.

[76] D. A. Jacobsohn, A. L. Gilman, A. Rademaker et al., "Evaluation of pentostatin in corticosteroid-refractory chronic graft-versus-host disease in children: a Pediatric Blood and Marrow Transplant Consortium study," Blood, vol. 114, no. 20, pp. 4354-4360, 2009.

[77] A. M. Alousi, D. J. Weisdorf, B. R. Logan et al., "Etanercept, mycophenolate, denileukin, or pentostatin plus corticosteroids for acute graft-versus-host disease: a randomized phase 2 trial from the Blood and Marrow Transplant Clinical Trials Network," Blood, vol. 114, no. 3, pp. 511-517, 2009. 


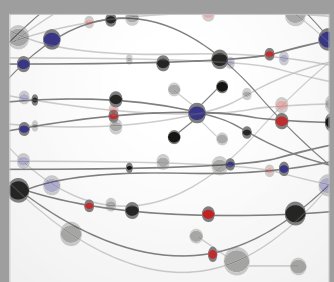

The Scientific World Journal
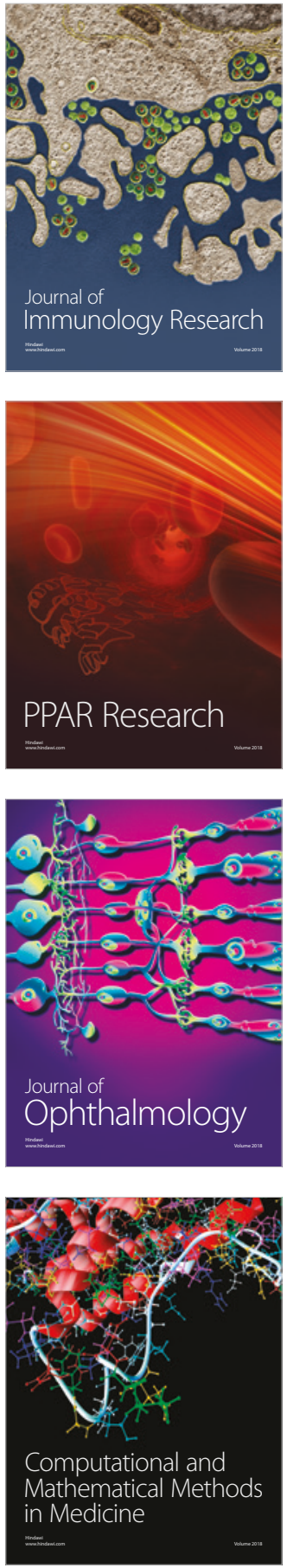

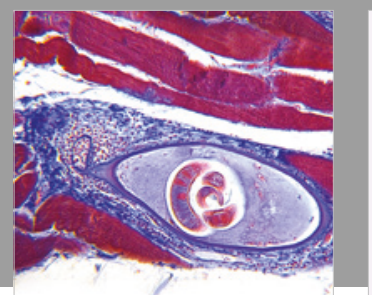

Gastroenterology Research and Practice

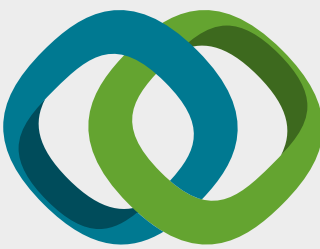

\section{Hindawi}

Submit your manuscripts at

www.hindawi.com
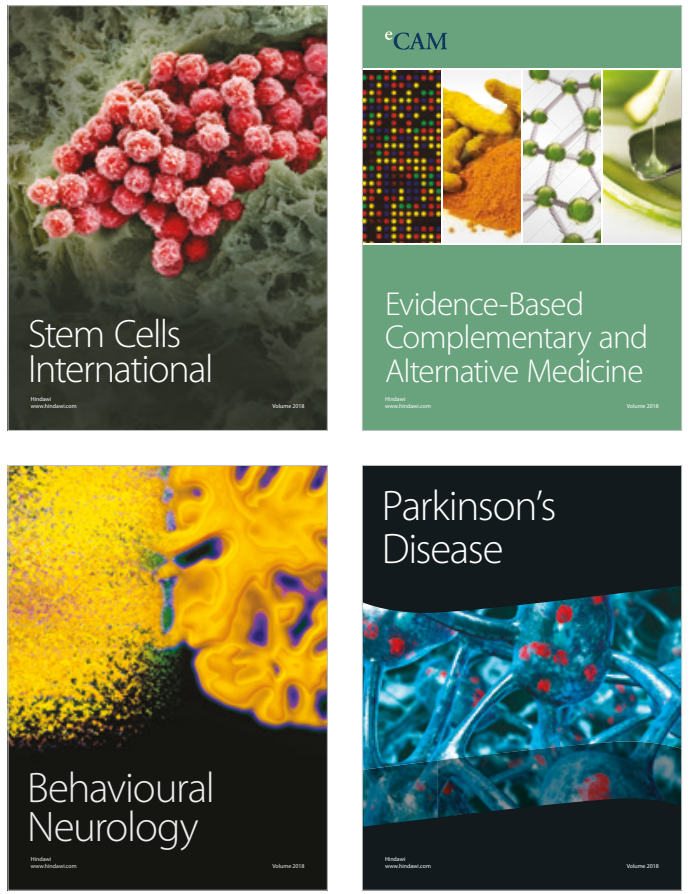

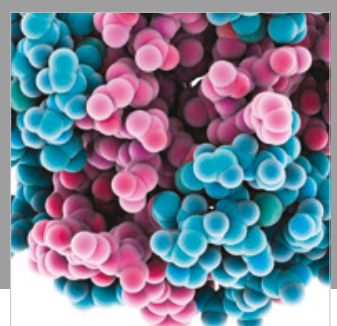

ournal of

Diabetes Research

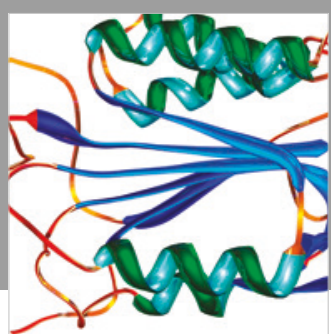

Disease Markers
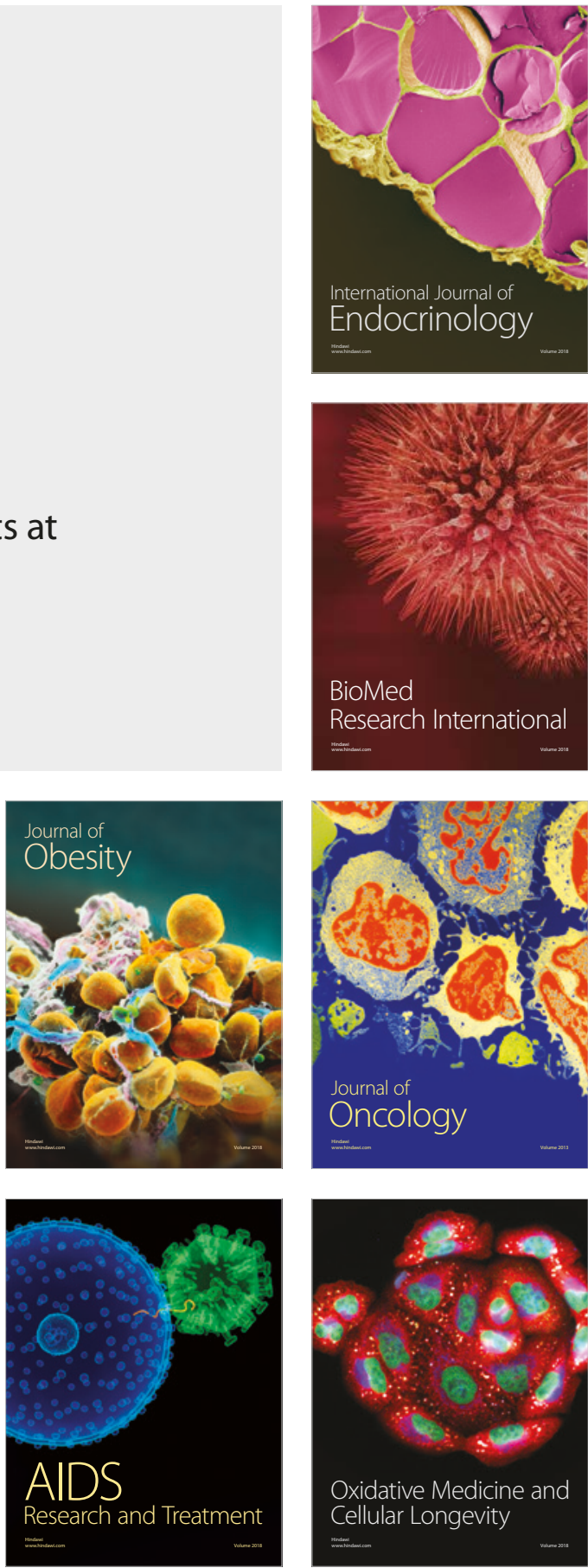